\title{
Draft genome of the Korean smelt Hypomesus nipponensis and its transcriptomic responses to heat stress in the liver and muscle
}

\author{
Biao Xuan, ${ }^{1,2}$ Jongbin Park, ${ }^{1,2}$ Sukjung Choi, ${ }^{2}$ Inhwan You, ${ }^{1,2}$ Bo-Hye Nam, ${ }^{3}$ Eun Soo Noh, ${ }^{3}$ Eun Mi Kim, ${ }^{3}$ Mi-Young Song, ${ }^{4}$ \\ Younhee Shin, ${ }^{5}$ Ji-Hyeon Jeon, ${ }^{5,6}$ and Eun Bae Kim (D) ${ }^{1,2, *}$ \\ ${ }^{1}$ Department of Applied Animal Science, College of Animal Life Sciences, Kangwon National University, Chuncheon 24341, Kangwon-do, Republic of Korea \\ ${ }^{2}$ Laboratory of Microbial Genomics and Big Data, College of Animal Life Sciences, Kangwon National University, Chuncheon 24341, Kangwon-do, Republic of Korea \\ ${ }^{3}$ Biotechnology Research Division, National Institute of Fisheries Science, Busan 46083, Republic of Korea \\ ${ }^{4}$ Inland Fisheries Research Institute, National Institute of Fisheries Science, Gapyeong 12453, Republic of Korea \\ ${ }^{5}$ Research and Development Center, Insilicogen Inc, Yongin 16954, Republic of Korea \\ ${ }^{6}$ Department of Biological Science, Sungkyunkwan University, Suwon 16419, Republic of Korea
}

*Corresponding author: Department of Applied Animal Science, College of Animal Life Sciences, Kangwon National University, Chuncheon 200-701, Republic of Korea. Email: itanimal@kangwon.ac.kr

\begin{abstract}
Pond smelt (Hypomesus nipponensis) is a cold-freshwater fish species and a winter economic aquaculture resource in South Korea. Because of its high susceptibility to abnormal water temperature from global warming, a large number of smelt die in hot summers. Here, we present the first draft genome of $\mathrm{H}$. nipponensis and transcriptomic changes in molecular mechanisms or intracellular responses under heat stress. We combined Illumina and PacBio sequencing technologies to generate the draft genome of $H$. nipponensis. Based on the reference genome, we conducted transcriptome analysis of liver and muscle tissues under normal (NT, $\left.5^{\circ} \mathrm{C}\right)$ vs. warm $\left(\mathrm{HT}, 23^{\circ} \mathrm{C}\right)$ conditions to identify heat stress-induced genes and gene categories. We observed a total of 1987 contigs with $\mathrm{N}_{50}$ of $0.46 \mathrm{Mbp}$, with the largest contig ( $3.03 \mathrm{Mbp}$ ) in the assembled genome. A total of 20,644 protein-coding genes were predicted, and 19,224 genes were functionally annotated: 15,955 genes for Gene Ontology terms and 11,560 genes for KEGG Orthology. We conducted the lost and gained genes analysis compared with three species that: human, zebrafish, and salmon. In the lost genes analysis, we detected that smelt lost 4461 (22.16\%), 2825 (10.62\%), and 1499 (3.09\%) genes compare with above three species, respectively. In the gained genes analysis, we observed that smelt gained 1133 (5.49\%), 1670 (8.09\%), and 229 (1.11\%) genes compared with the above species, respectively. From transcriptome analysis, a total of 297 and 331 differentially expressed genes (DEGs) with a false discovery rate $<0.05$ were identified in the liver and muscle tissues, respectively. Gene enrichment analysis of DEGs indicates that upregulated genes were significantly enriched for lipid biosynthetic process (GO:0008610, $P<0.001)$ and regulation of apoptotic process (GO:0042981, $P<0.01$ ), and genes were downregulated by immune responses such as myeloid cell differentiation (GO:0030099, $P<0.001)$ in the liver under heat stress. In muscle tissue, upregulated genes were enriched for hypoxia (GO:0001666, $P<0.05)$, transcription regulator activity (GO:0140110, $P<0.001)$, and calcium-release channel activity (GO:0015278, $P<0.01)$, and genes were downregulated for a nicotinamide nucleotide biosynthetic process (GO:0019359, $P<0.01$ ). The results of KEGG pathway analysis were similar to that of gene enrichment analysis. The draft genome and transcriptomic of $H$. nipponensis will be a useful genetic resource for functional and evolutionary studies. Our findings will improve understanding of molecular mechanisms and heat responses and be useful for predicting survival of the smelt and its closely related species under global warming.
\end{abstract}

Keywords: Hypomesus nipponensis; genome; transcriptome; high temperature; heat stress

\section{Introduction}

Pond smelt (Hypomesus nipponensis) is a member of the family Osmeridae and native to several countries, such as South Korea, China, Japan, and the United States. Smelt, a cold-freshwater fish species, it is called "Bing eo" in Korea, which means ice-fish, because it resides in low-temperature water $\left(0-15^{\circ} \mathrm{C}\right)$. It popular as a winter fishing species and as food in Korea, so many smelt festivals are held in winter. Smelt is an anadromous species (Saruwatari et al. 1997), but now it is cultivated in many reservoirs in South Korea. In a previous study, smelt samples from South Korea were identified as H. nipponensis (Choi and Kim 2019).
Abnormal increases in temperature lead to the death of many cold-water fish species. Therefore, many studies have focused on acute and chronic heat stress on fish (Logan and Buckley 2015). In Korea, there was local news report that the death of smelt in hot summers brings serious harm to the smelt industry. Therefore, understanding the characteristics of smelt will greatly improve responses to the problems faced. That said, however, there is no research on the genome or transcriptome of $\mathrm{H}$. nipponensis. Furthermore, transcriptome analysis provides useful insight into the specific and general response to water temperature, such as heat shock response (Narum and Campbell 
2015). In addition, the cellular response of different smelt species to high water temperature is different. In longfin smelt, many differentially expressed genes (DEGs) associated with heat shock proteins and chaperones are upregulated generally in fish responding to heat stress. Conversely, in Delta smelt, DEGs are associated with protein synthesis and metabolic processes (Basu et al. 2002). Cells generally regulate their own genes to survive under stress; however, occasionally they start programming cell death to induce apoptosis (Fulda et al. 2010). Increasing water temperature has many effects on fish, such as oxidative stress, endoplasmic reticulum (ER) stress, decreased immune function, hypoxia, and reproductive dysfunction that affects egg production and fertility (Olsvik et al. 2013; Lu et al. 2016; Qiang et al. 2017). Because specific tissue responses differ under heat stress, many heat stress-related studies used different tissues of fish, such as the liver, muscle, heart, kidney, brain, and gill (Logan and Buckley 2015). For example, ER stress was identified in Atlantic salmon liver under heat stress, where it promoted cell repair and reduced unfolded proteins; however, excessive ER stress led to cell apoptosis (Shi et al. 2019). ER stress also occurs in heat stress-affected rainbow trout (Oncorhynchus mykiss), where it is accompanied by changes in the immune system and post-transcriptional regulation of spliceosome (Huang et al. 2018). In teleost coho salmon (Oncorhynchus kisutch) liver, severe heat stressors can affect the redox state and induce oxidative stress (Nakano et al. 2014). Moreover, water temperature affects the concentration of dissolved oxygen in water, and low oxygen concentration in water is fatal to fish, leading to hypoxia. Dissolved oxygen plays an important role in maintaining biochemical and physiological processes. Hypoxia may limit energy processes and adversely affect growth, reproduction, and survival (Kelly et al. 2020). Many factors affect the growth and survival of fish; therefore, it is necessary to clarify the mechanism of heat stress responses of smelt to reduce their mortality effectively.

In the present study, we used sequencing technology to analyze the whole genome of $\mathrm{H}$. nipponensis and transcriptome analysis to compare gene expression to understand the intracellular response mechanism of $\mathrm{H}$. nipponensis at different temperatures in the liver and muscle tissues.

\section{Materials and Methods Ethics statement}

All the procedures performed on animals were approved by the Institutional Animal Care and Use Committee (accept number: KW-181109-2) at Kangwon National University.

\section{Source of $\mathrm{H}$. nipponensis and genome sequencing}

Whole-genome sequencing was conducted for three samples of smelt from Inje narincheon (South Korea, 38 $04^{\prime} 34.0^{\prime \prime} \mathrm{N} ; 128^{\circ} 11^{\prime}$ $17.2^{\prime \prime} \mathrm{E}$ ), and the genomic DNA was extracted from the whole bodies using the DNeasy ${ }^{\circledR}$ Blood \& Tissue kit (Qiagen, GmbH, Hilden, Germany). Illumina libraries for whole bodies were constructed using the TruSeq Nano Sample Prep kit (Illumina, San Diego, CA, USA) following the manufacturer's instructions. A PacBio Sequel platform library was constructed using the PacBio Template Prep kit. PacBio-generated reads were used for de novo assembly, and Illumina paired-end reads were mapped to the draft genome assembly to error correct by pilon v.1.23 (Walker et al. 2014). De novo assembly with PacBio reads was conducted by FALCON-UNZIP software version 1.2.5 using the parameters length_cutoff $=13 \mathrm{~kb}$ and length_cutoff_pr $=10 \mathrm{~kb}$. H. nipponensis genome size was estimated using k-mer (17-, 19-, and 21-mers) analysis with Jellyfish 2.1 .3 software. The assembly quality was assessed using Benchmarking Universal Single Copy Orthologs
(BUSCO) version 3.0 (Simão et al. 2015). Supplementary Figure S1 shows the analysis workflow of the fish genome.

\section{PacBio (Iso-Seq) sequencing}

Total RNA was isolated from liver and muscle tissues. For PacBio Iso-Seq data, we randomly selected 12 samples from the transcriptome analysis group (six liver and six muscle samples). The total RNA of the 12 samples was pooled for sequencing. The Iso-Seq library was prepared using a SMATer PCR cDNA Synthesis kit. We used Trinity (Haas et al. 2013) software to perform a genomeguided assembly and combine Iso-Seq data to predict the gene model.

\section{H. nipponensis genome functional annotation}

The predicted genes were subjected to search against the National Center for Biotechnology Information (NCBI) nonredundant protein database (20190306 ver.) using BLASTx, with an evalue cutoff of $1 \mathrm{E}^{-3}$. Gene name and description were assigned to each gene based on the highest hit with BLASTx. The genes were submitted to the Gene Ontology (GO) and Kyoto Encyclopedia of Genes and Genomes (KEGG) databases to obtain the functional category and pathway information using Blast2GO and KAAS (Moriya et al. 2007), respectively.

\section{Temperature trial and tissue sampling for RNA sequencing}

Smelt were caught in the Hwacheon Dam (South Korea, 38 $07^{\prime}$ $01.1^{\prime \prime} \mathrm{N} ; 127^{\circ} 46^{\prime} 43.1^{\prime \prime} \mathrm{E}$ ), and 100 fish were randomly placed in two 20-L tanks (50 fish per tank, NT and HT groups). The fish were fed, and acclimatization occurred for $2 \mathrm{~h}$ at a water (from Hwacheon Dam) temperature of $5^{\circ} \mathrm{C}$. After acclimation, to simulate temperature conditions in their natural environment, one tank was heated from $5^{\circ} \mathrm{C}$ to $23^{\circ} \mathrm{C}$ at a constant rate of $4.5^{\circ} \mathrm{C}$ per $\mathrm{h}$ by hotrod, while the other tank was maintained at the same temperature $\left(5^{\circ} \mathrm{C}\right)$ with ice packs. Oxygen was supplied throughout the experiment. Sampling began until loss of equilibrium among the fish population appeared in the HT group $\left(23^{\circ} \mathrm{C}\right)$; however, no loss of equilibrium among the fish population was observed in the NT group $\left(5^{\circ} \mathrm{C}\right)$. A total of 8 female fish were sampled (4 active and 4 inanimate from the NT and HT groups, respectively), and a total of 12 male fish were sampled ( 6 active and 6 inanimate from the NT and HT groups, respectively). After being sacrificed, muscle and liver tissues were immediately frozen in liquid nitrogen for gene expression profiling analysis.

\section{Constructing mRNA libraries and sequencing}

Tissue mRNA was extracted from the liver and muscle samples using TRIzol ${ }^{\circledR}$ reagent (Thermo Fisher Scientific, Waltham, MA, USA) and purified to remove DNA contamination using the TURBO DNA-free ${ }^{\mathrm{TM}}$ kit (Invitrogen, Carlsbad, CA, USA). RNA concentration and quality were determined using an Epoch Microplate Spectrophotometer (BioTek, Winooski, VT, USA). The 37 sequencing libraries were created by reverse-transcription from $2 \mu \mathrm{g}$ RNA from each sample using the TruSeq Stranded mRNA Library Prep kit (Illumina, San Diego, CA, USA). Index adapters were added to identify sequences for each sample in the final data. Subsequently, the 37 libraries were subjected to paired-end $(2 \times 101 \mathrm{bp})$ sequencing on the Illumina NovaSeq6000 Sequencing system.

\section{Detection of lost and gained genes}

To detect lost and gained genes in the Korean smelt genome, we compared their fragmented sequences (raw reads, assembled 
DNA contigs, and assembled RNA transcripts) with several reference genomes: human (Homo sapiens), zebrafish (Danio rerio), and Atlantic salmon (Salmo salar). Sequence alignment was conducted by GASSST (v1.28) software. Supplementary Figure S2 shows the method by which sequence alignment coverage (\%) was calculated for each gene from the alignment. A low alignment coverage $(<10 \%)$ indicates the absence of a gene in the genome.

\section{Data analysis of mRNA}

After removing the reads containing adaptor contamination using Trimmomatic v0.36, the clean reads were mapped to the assembled reference $H$. nipponensis genome using Bowtie v2.2.3. RSEM v1.2.31 was used to quantify gene abundances according to each $H$. nipponensis gene. Analysis of genes differentially expressed in the NT and HT groups was performed using the edgeR package (Robinson et al. 2010), and a false discovery rate (FDR) $<0.05$ was considered a threshold to identify significantly DEGs. GO enrichment analysis and KEGG statistical enrichment analysis of DEGs were performed using in-house Perl scripts (parent-child method) and the clusterProfiler R package (Yu et al. 2012), respectively. The significantly enriched GO terms were determined by Fisher's exact test, with a $P<0.05$, and KEGG pathways were determined using FDR $<0.05$.

\section{Results \\ Genome sequence data and genome size estimation}

Using the PacBio long-read sequencing approach, a $126 \times$ coverage (58.60 Gbp) of $\mathrm{H}$. nipponensis genome was obtained from $>20$ $\mathrm{kb}$ libraries. The short-read libraries (Illumina) generated an $80 \times$ coverage (36.99 Gbp) of genome, obtained from paired-end sequencing $(2 \times 101 \mathrm{bp})$ (Supplementary Table S1). The genome size was estimated to be $464 \mathrm{Mb}$ using $\mathrm{k}$-mer $(k=17)$ analysis (Supplementary Table S2 and Figure S3).

\section{Iso-Seq data generation}

Isoform sequencing (PacBio) generated $0.49 \mathrm{~Gb}$ of data, which included 477,478 circular consensus sequences (CCS) reads and a mean 1023 CCS read length, yielding 18,799 transcripts (@Supplementary Table S3), which were used to generate the assistant file for $a b$ initio gene model prediction.

\section{De novo assembly of genome and quality assessment}

Using long-read sequences from PacBio, the primary genome assembly was $631.85 \mathrm{Mbp}$ in size, with 4106 contigs (largest contig of 3.03 Mbp, $\mathrm{N}_{50}$ of $0.32 \mathrm{Mbp}$, and $\mathrm{L}_{50}$ of 477). Evaluation of the genome completeness analysis against the 4584 genes of Actinopterygii BUSCO result showed that the 4290 genes (93.6\%) were completely retrieved in the assembled genome, including 3911 single-copy genes (85.3\%) and 379 duplicated genes (8.3\%). In addition, 84 fragmented genes (1.8\%) and 210 missing genes (4.5\%) were found (Supplementary Table S4). After error correction using pilon, the final improved primary genome assembly of 1987 contigs showed a total size of $499.59 \mathrm{Mbp}$, with an $\mathrm{N}_{50}$ of $0.46 \mathrm{Mbp}, \mathrm{L}_{50}$ of 300 contigs, GC content of $45.64 \%$, and the largest contig length of $3.03 \mathrm{Mbp}$ (Supplementary Table S5). Supplementary Tables S6 and S7 show the Illumina and PacBio sequencing statistics. Table 1 shows the $H$. nipponensis draft genome statistics.
Table 1 Hypomesus nipponensis draft genome statistics

\begin{tabular}{lc}
\hline Index & H. nipponensis \\
\hline Number of contigs & 1,987 \\
Total contig length & $498,930,205$ \\
Estimated genome size & $464,167,205$ \\
Total length/estimated genome size & $107.48 \%$ \\
Minimum contig length & 19,619 \\
Maximum contig length & $3,031,274$ \\
Average contig length & 251,097 \\
Contig length N50 & 464,523 \\
GC contents & $45.63 \%$ \\
BUSCO (Actinopterygii) complete & $93.58 \%$ \\
BUSCO (Vertebrata) complete & $95.32 \%$ \\
CEGMA complete & $92.74 \%$ \\
\hline
\end{tabular}

\section{Gene prediction and functional annotation}

Evidence-based $a b$ initio gene predictions with Augustus predicted 20,644 protein-coding genes. Among these, $93.12 \%(19,224)$ of predicted genes were annotated using the nonredundant database, while 6.88\% (1420) of genes remained unannotated (Supplementary Table S8). Figure 1A shows the Blast top 10 hit species. Analysis of GO terms revealed that they were assigned to 15,955 genes on the three primary categories of ontology [biological process (BP), cellular component (CC), and molecular function (MF)]. Figure 1B shows the top 10 assigned GO terms for each category. A total of 11,560 genes were annotated with 7 categories on 447 pathways. Figure 1C shows the top three assigned KEGG terms for each category. Table 2 shows the $H$. nipponensis consensus gene model.

\section{Genome comparison for detection of lost and gained genes}

We compared the genome of H. nipponensis with that of other species, including H. sapiens, D. rerio, and S. salar, for detection of lost and gained genes. For lost genes found from the alignment of the $H$. nipponensis genome compared with those of the other species, we compared 20,129, 26,612, and 48,548 genes in $H$. sapiens, D. rerio, and S. salar, respectively. We detected 4461 (22.16\%), 2825 (10.62\%), and $1499(3.09 \%)$ genes with coverage less than 10\% and 6047 (30.04\%), 3901 (14.66\%), and 2008 (4.14\%) genes with coverage less than 15\% (Supplementary Table S9). For gained genes found from the alignment of 20,644 genes of H. nipponensis compared with those of the other species, we observed $1133(5.49 \%)$, $1670(8.09 \%)$, and $229(1.11 \%)$ genes with coverage less than 10\% and 1911 (9.26\%), 2484 (12.03\%), and 362 (1.75\%) genes with coverage less than 15\% (Supplementary Table S10).

\section{GO enrichment of lost and gained genes}

Gene enrichment analysis comparing smelt with human showed that smelt lost some genes involved in stimulus, reproduction, nucleotide, and metal ions. Comparison with salmon showed that smelt lost chemotaxis-, immune system-, lipid-, mitochondria-, neuron-, nucleotide-, and reproduction-related genes (Supplementary Table S11). In gained genes enrichment analysis, compared with human, smelt gained chemotaxis-, heparin production-, immune system-, and stimulation-related genes. Compared with zebrafish and salmon, smelt gained chemotaxisand immune system-related genes, respectively (Supplementary Table S12).

\section{RNA-seq of liver and muscle transcriptome profiles at different temperature conditions}

To identify differences in gene expression in smelt liver and muscle tissues at different temperatures, we constructed and sequenced 20 

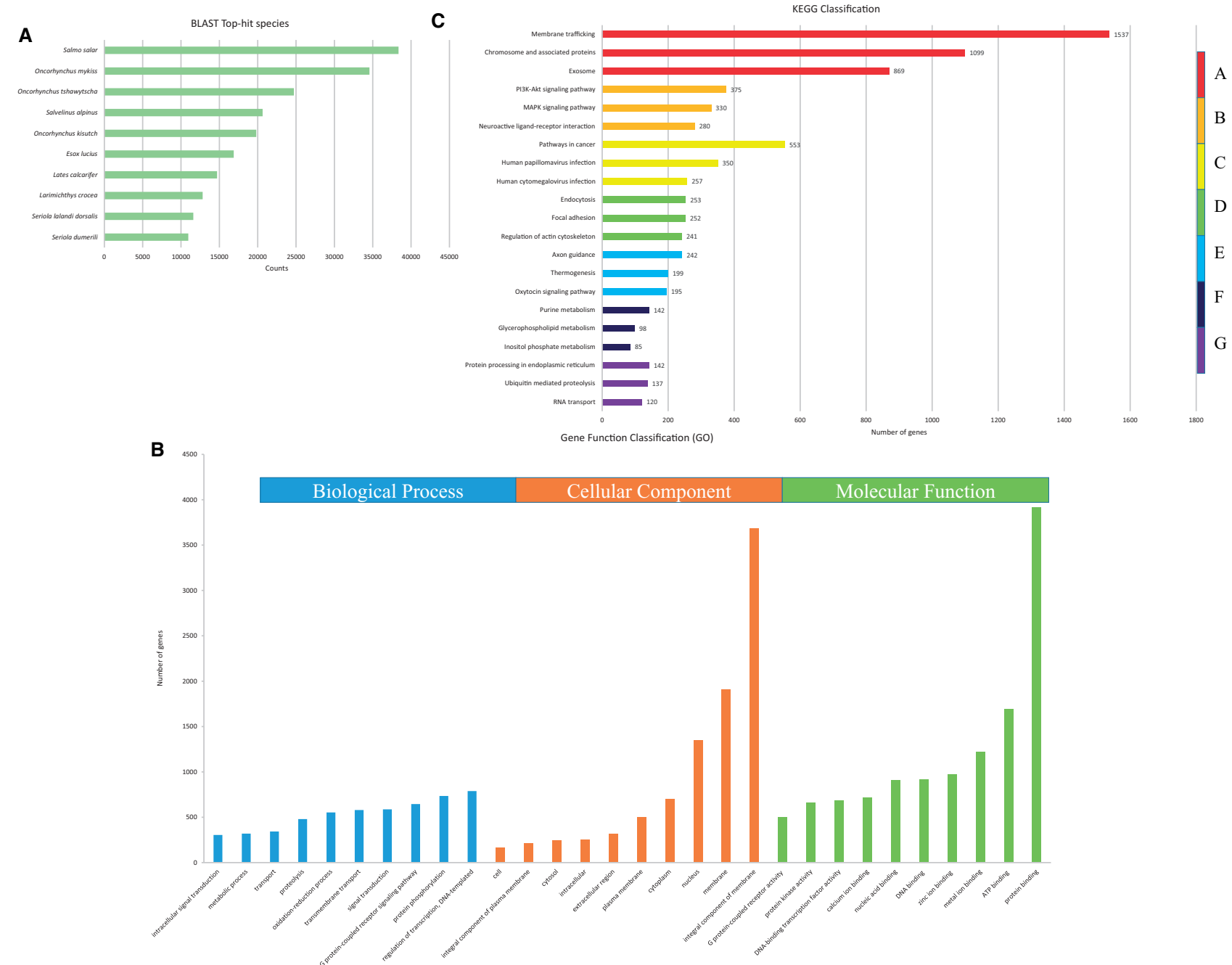

Figure 1 Annotation and functional classification of unigenes in the genome of H. nipponensis. (A) Blast top 10 hit species. (B) GO analysis was performed at level 2 for the three main categories (BP, CC, and MF). (C) Pathway assignment based on the KEGG database. Unigenes were classified into seven main categories (A: Genetic Information Processing; B: Metabolism; C: Organismal Systems; D: Cellular Processes; E: Human Diseases; F: Environmental Information Processing; G: Brite Hierarchies).

Table $2 \mathrm{H}$. nipponensis consensus gene model

\begin{tabular}{lll}
\hline Category & \multicolumn{1}{c}{ Index } & \multicolumn{1}{c}{ Value } \\
\hline Gene & Gene count & 20,644 \\
& Maximum gene length & 207,358 (HYNIP00072CG0010) \\
& Minimum gene length & 222 (HYNIPO0473CG0050) \\
& Average gene length & $12,073.93$ \\
& Total gene length & $249,254,225$ \\
& Genome coverage & $49.96 \%$ \\
Exon & Exon count & 193,124 \\
& Exon count per gene & 9.35 \\
& Average exon length & 178 \\
& Total exon length & $34,376,477$ \\
Intron & Genome coverage & $6.89 \%$ \\
& Intron count & 172,480 \\
& Average intron length & $1,245.81$ \\
& Total intron length & $214,877,748$ \\
& Genome coverage & $43.07 \%$ \\
\hline
\end{tabular}

cDNA libraries on the Illumina NovaSeq6000 platform with pair-end sequencing. The clean reads were generated by filtering the raw reads from the NT and HT groups. Supplementary Tables S13 and S14 show the statistics of clean reads filtered from raw reads, and the mean quality is more than $30 \%$ for each sample.

\section{Gene expression profiling}

A total of 297 and 331 genes were significantly differentially expressed in liver and muscle tissues of female fish in the HT group compared with those in the NT group, respectively [|log2(Fold change) $\mid>1$, FDR < 0.05]. In the liver tissue of female fish, 297 DEGs, including 154 upregulated DEGs and 143 downregulated DEGs, were identified. In the muscle tissue of female fish, 331 DEGs, including 192 upregulated DEGs and 139 downregulated DEGs, were identified. In the liver tissue of male fish, eight DEGs, including four upregulated DEGs and four downregulated DEGs, were identified. In the muscle tissue of male fish, 29 DEGs, including 9 upregulated and 20 downregulated DEGs, were identified (Figure 2). The volcano plot showed the DEG analysis in the liver and muscle tissues of male fish. Because the number of DEGs in tissue from male fish is significantly less than that in tissue from female fish, the following gene functional enrichment analysis involves only tissue from female fish. Supplementary Table 16 shows all DEGs.

\section{GO analysis of DEGs in the liver and muscle}

In enrichment analysis, DEGs were enriched GO terms (threshold as $\mathrm{P}<0.05$ and odds ratio $>1$ ). For the liver, 128 upregulated and 
A

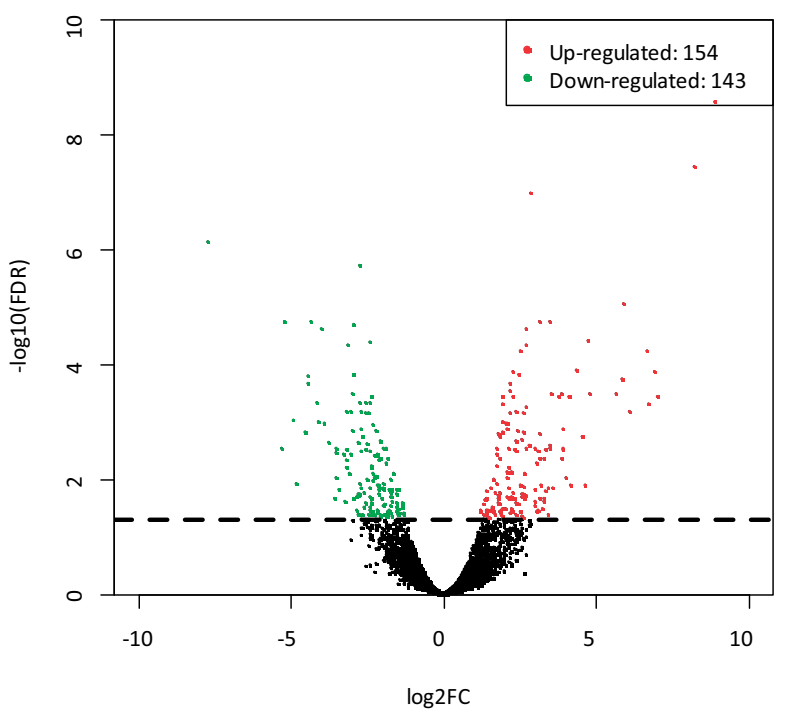

C

HT vs NT (Male Liver)

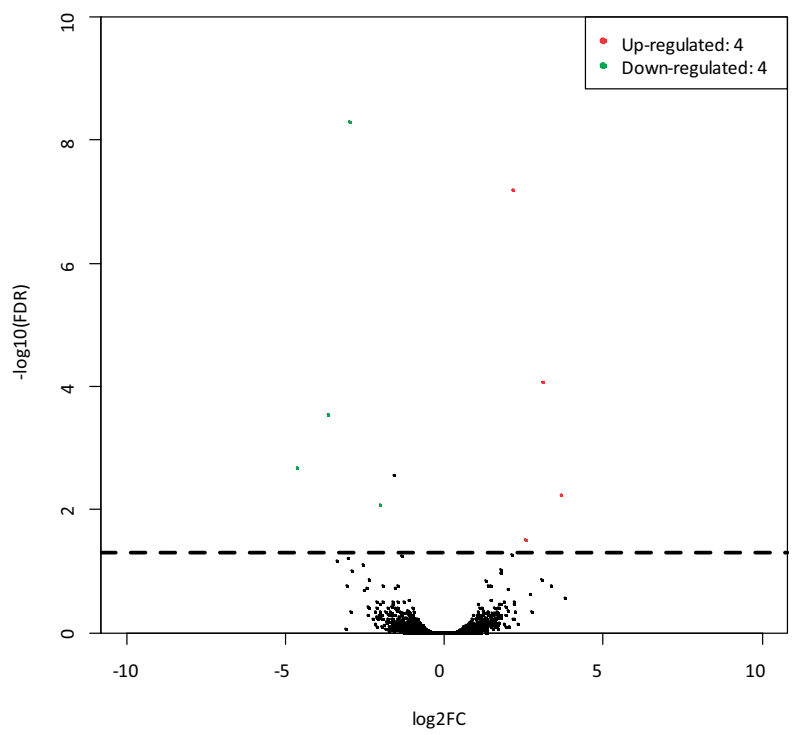

B

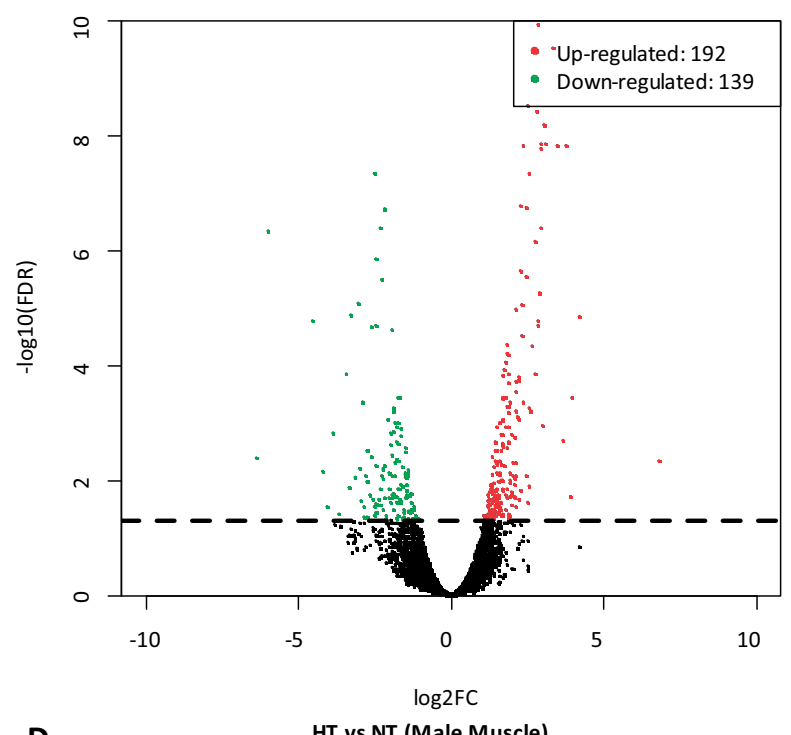

D

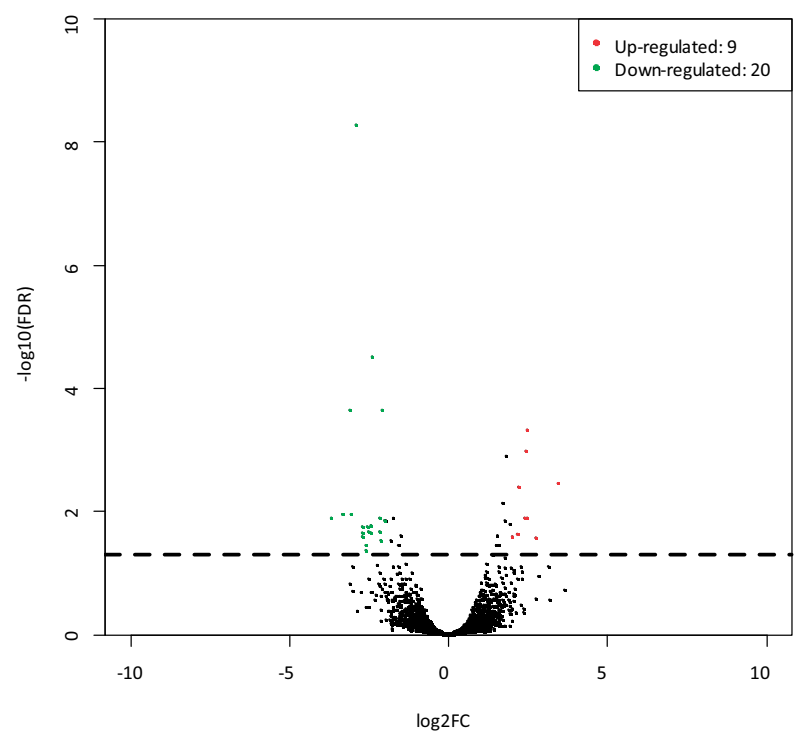

Figure 2 Differential expression of high temperature in H. nipponensis us. low temperature. (A) DEGs in the liver of female fish. (B) DEGs in muscle of female fish. (C) DEGs in the liver of male fish. (D) DEGs in muscle of male fish. The dots above the dotted line are FDR $<0.05,|\log 2 \mathrm{FC}| \geq 1$.

115 downregulated DEGs were annotated with GO terms. Upregulated GO terms included lipid metabolism-, cell death-, DNA binding-, and stress-related genes, and downregulated GO terms included immune system-, membrane protein-, and digestion-related genes (Table 3). For the muscle, 161 upregulated and 114 downregulated DEGs were annotated with GO terms. Upregulated GO terms included apoptosis-, hypoxia-, ion transportation-, transcription-, and component organization-related genes, and downregulated GO terms included nicotinamide nucleotide- and ubiquitination-related genes (Table 4).

\section{KEGG pathway enrichment analysis of DEGs in the liver and muscle}

Four KEGG pathways were enriched in the liver tissue. One pathway (FDR < 0.1), including the terpenoid backbone biosynthesis pathway, was upregulated, while three pathways (FDR $<0.05$ ), including the influenza A, Jak-STAT signaling, and pancreatic secretion pathways, were downregulated. Thirty-two KEGG pathways were enriched in the muscle tissue. Mitogen-activated protein kinase $(\mathrm{MAPK})$ signaling $(\mathrm{FDR}<0.05)$, oxytocin signaling pathways (FDR $<0.05)$, and HIF-1 signaling pathway (FDR $<0.05)$ were upregulated, while four pathways (FDR $<0.05$ ), including inflammatory bowel disease (IBD), Leishmaniasis, African trypanosomiasis, and type 1 diabetes pathways, were downregulated (Figure 3).

\section{Discussion}

H. nipponensis is one of the most popular freshwater fish species in South Korean winters. With the rise in global warming, fish are suffering from heat stress, and a large number of deaths have been observed, resulting in great economic losses to the 
Table 3 Gene enrichment analysis of DEGs in liver tissue under heat stress

\begin{tabular}{|c|c|c|c|c|c|c|c|c|c|}
\hline & Category & GO ID & GO term & Bg_Gene & $\begin{array}{l}\mathrm{Tg}_{-} \\
\text {Gene }\end{array}$ & $\begin{array}{l}\mathrm{Bg}_{-} \\
\text {Gene }\end{array}$ & $\begin{array}{l}\mathrm{Tg}_{-} \\
\text {Gene }\end{array}$ & $\begin{array}{l}P- \\
\text { value }\end{array}$ & $\begin{array}{l}\text { Odds } \\
\text { ratio }\end{array}$ \\
\hline \multirow[t]{12}{*}{ Upregulated } & \multirow[t]{5}{*}{ Lipid metabolism } & GO:0008610 & Lipid biosynthetic process & 15,955 & 128 & 127 & 7 & 0.00 & 6.87 \\
\hline & & GO:0006629 & Lipid metabolic process & 15,955 & 128 & 371 & 10 & 0.00 & 3.36 \\
\hline & & GO:0008299 & Isoprenoid biosynthetic process & 15,955 & 128 & 13 & 3 & 0.00 & 28.74 \\
\hline & & GO:0006721 & Terpenoid metabolic process & 15,955 & 128 & 16 & 2 & 0.01 & 15.57 \\
\hline & & GO:0008202 & Steroid metabolic process & 15,955 & 128 & 35 & 4 & 0.00 & 14.24 \\
\hline & \multirow[t]{3}{*}{ Cell death } & GO:0042981 & Regulation of apoptotic process & 15,955 & 128 & 154 & 6 & 0.00 & 4.86 \\
\hline & & GO:0043067 & Regulation of programmed cell death & 15,955 & 128 & 157 & 6 & 0.00 & 4.76 \\
\hline & & GO:0010941 & Regulation of cell death & 15,955 & 128 & 164 & 6 & 0.00 & 4.56 \\
\hline & \multirow[t]{3}{*}{ DNA binding } & GO:0030983 & Mismatched DNA binding & 15,955 & 128 & 13 & 2 & 0.01 & 19.16 \\
\hline & & GO:0006298 & Mismatch repair & 15,955 & 128 & 15 & 2 & 0.01 & 16.61 \\
\hline & & GO:0003690 & Double-stranded DNA binding & 15,955 & 128 & 117 & 4 & 0.02 & 4.26 \\
\hline & Stress & GO:0080135 & Regulation of cellular response to stress & 15,955 & 128 & 49 & 3 & 0.01 & 7.63 \\
\hline & \multirow[t]{2}{*}{ Membrane protein } & GO:0005834 & Heterotrimeric G-protein complex & 15,955 & 115 & 30 & 3 & 0.00 & 13.87 \\
\hline & & GO:1905360 & GTPase complex & 15,955 & 115 & 30 & 3 & 0.00 & 13.87 \\
\hline & Digestion & GO:0007586 & Digestion & 15,955 & 115 & 13 & 2 & 0.01 & 21.33 \\
\hline
\end{tabular}

Table 4 Gene enrichment analysis of DEGs in muscle tissue under heat stress

\begin{tabular}{|c|c|c|c|c|c|c|c|c|c|}
\hline & Category & GO ID & GO term & $\begin{array}{l}\mathrm{Bg}_{-} \\
\text {Gene }\end{array}$ & $\begin{array}{l}\mathrm{Tg}_{-} \\
\text {Gene }\end{array}$ & $\begin{array}{l}\mathrm{Bg}_{-} \\
\text {Gene }\end{array}$ & $\begin{array}{c}\mathrm{Tg} \\
\text { _Gene }\end{array}$ & $\begin{array}{l}P \text { - } \\
\text { value }\end{array}$ & $\begin{array}{l}\text { Odds } \\
\text { ratio }\end{array}$ \\
\hline \multirow[t]{11}{*}{ Upregulated } & \multirow[t]{3}{*}{ Apoptosis } & GO:0097190 & Apoptotic signaling pathway & 15,955 & 161 & 16 & 3 & 0.00 & 18.56 \\
\hline & & GO:0008630 & $\begin{array}{l}\text { Intrinsic apoptotic signaling pathway in } \\
\text { response to DNA damage }\end{array}$ & 15,955 & 161 & 8 & 2 & 0.00 & 24.74 \\
\hline & & GO:0097193 & Intrinsic apoptotic signaling pathway & 15,955 & 161 & 13 & 2 & 0.01 & 15.24 \\
\hline & \multirow[t]{2}{*}{ Hypoxia } & GO:0036293 & Response to decreased oxygen levels & 15,955 & 161 & 17 & 2 & 0.02 & 11.65 \\
\hline & & GO:0001666 & Response to hypoxia & 15,955 & 161 & 17 & 2 & 0.02 & 11.65 \\
\hline & Ion transportation & GO:0019722 & Calcium-mediated signaling & 15,955 & 161 & 15 & 2 & 0.01 & 13.20 \\
\hline & \multirow[t]{4}{*}{ Transcription } & GO:0003700 & DNA-binding transcription factor activity & 15,955 & 161 & 525 & 17 & 0.00 & 3.21 \\
\hline & & GO:0140110 & Transcription regulator activity & 15,955 & 161 & 608 & 18 & 0.00 & 2.93 \\
\hline & & GO::0000981 & $\begin{array}{l}\text { DNA-binding transcription factor activity, } \\
\text { RNA polymerase II-specific }\end{array}$ & 15,955 & 161 & 102 & 5 & 0.00 & 4.86 \\
\hline & & GO:0006355 & Regulation of transcription, DNA-templated & 15,955 & 161 & 1,038 & 21 & 0.00 & 2.00 \\
\hline & $\begin{array}{l}\text { Component } \\
\text { organization }\end{array}$ & GO:0051639 & Actin filament network formation & 15,955 & 161 & 2 & 2 & 0.00 & 98.80 \\
\hline \multirow{7}{*}{ Downregulated } & \multirow{5}{*}{$\begin{array}{l}\text { Nicotinamide } \\
\text { nucleotide }\end{array}$} & GO:0046496 & Nicotinamide nucleotide metabolic process & 15,955 & 114 & 15 & 2 & 0.01 & 18.65 \\
\hline & & GO:0019363 & Pyridine nucleotide biosynthetic process & 15,955 & 114 & 15 & 3 & 0.00 & 27.93 \\
\hline & & GO:0019362 & Pyridine nucleotide metabolic process & 15,955 & 114 & 16 & 3 & 0.00 & 26.19 \\
\hline & & GO:0072525 & Pyridine-containing compound biosynthetic process & 15,955 & 114 & 18 & 3 & 0.00 & 23.29 \\
\hline & & GO:0072524 & Pyridine-containing compound metabolic process & 15,955 & 114 & 19 & 3 & 0.00 & 22.07 \\
\hline & \multirow[t]{2}{*}{ Ubiquitination } & GO:0061630 & Ubiquitin protein ligase activity & 15,955 & 114 & 73 & 3 & 0.02 & 5.75 \\
\hline & & GO:0061659 & Ubiquitin-like protein ligase activity & 15,955 & 114 & 73 & 3 & 0.02 & 5.75 \\
\hline
\end{tabular}

aquaculture industry. The present study reports the first highquality draft genome assembly of $H$. nipponensis from the family Osmeridae (order Osmeriformes) and the analysis of transcriptome in heat stress to elucidate the mechanism of cellular response in liver and muscle tissues.

\section{Genome sequencing}

We successfully generated $H$. nipponensis draft genome assembly of 1987 highly contiguous contigs (498.3 Mbp) with a high $\mathrm{N}_{50}$ (0.47 Mbp). The estimated genome size of $464 \mathrm{Mbp}$ was consistent with that of another member of Osmeridae and greater than that of Osmerus eperlanus (European smelt, $342.8 \mathrm{Mbp}$ ) in the family Osmeridae (Malmstrøm et al. 2016). Compared with other species (mammal, bird, bony fish, and cartilaginous fish), the genome size of smelt is smaller than that of other species, but the GC content of genome and coding sequence (CDS) and GC content of CDS are similar to those of other species, and the number of CDS is even more than that of other species (Supplementary Table $\mathrm{S15}$ ). The genome size of $\mathrm{H}$. nipponensis is within the range of most published fish genome sizes (Fan et al. 2020). 

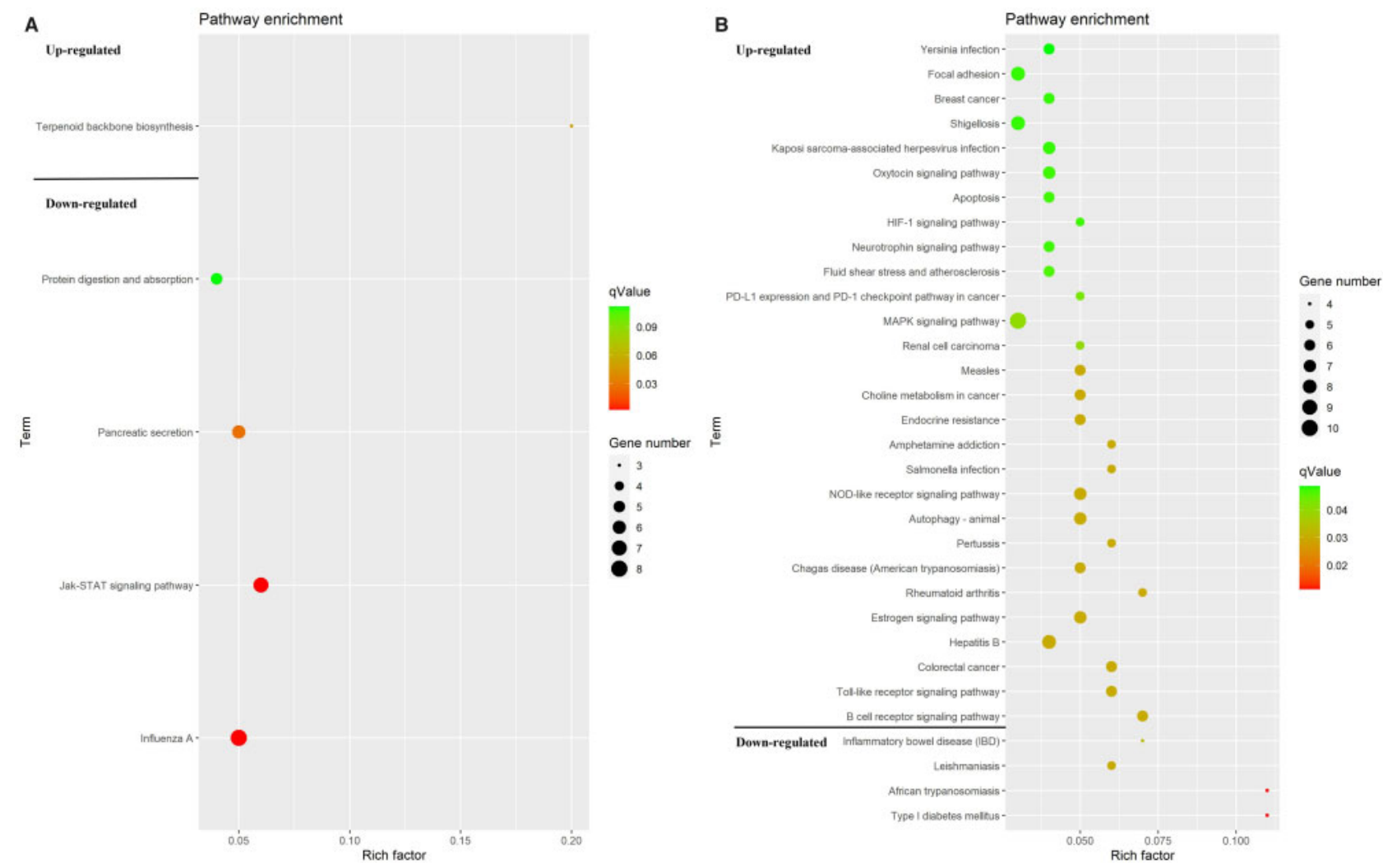

Figure 3 An overview of the KEGG pathway significantly enriched in DEGs in the (A) liver, and (B) muscle. The specific pathways are plotted along the yaxis, and the $\mathrm{x}$-axis indicates the enrichment factor. The colored dots indicate the number of significant DEGs associated with each corresponding pathway: pathways with larger-sized dots contain a higher number of genes. The color of the each dot indicates the corrected P-value for the corresponding pathway.

\section{Lost and gained genes}

In the lost gene analysis, we compared $H$. nipponensis with human (H. sapiens), zebrafish (D. rerio), and Atlantic salmon (S. salar). The lowest number of lost genes was observed when comparing $H$. nipponensis with S. salar because salmon is a cold-water species that lives in similar water temperature. In addition, these two species are both anadromous; also, Osmeriformes are close relatives of the Salmoniformes. One of smelt species that rainbow smelt (Osmerus mordax) has high levels of similarity (86\%) to salmonid genes (Von Schalburg et al. 2008). In the lost gene enrichment analysis, compared with human, smelt may not sense bitter taste because smelt eat phytoplankton; they may lose their taste buds for bitterness to survive. In one study comparing the naked mole rat (NMR) and human genomes, NMRs lost their receptors for bitter taste because NMRs live underground and their staple food is plant roots, most of which have a bitter taste, which facilitates this evolution (Kim et al. 2011). Moreover, the spermatogenesis of smelt may be different from that of human. With evolution, the genes involved in sex- and reproduction-related genes, such as mating behavior, fertilization, spermatogenesis, and sex determination, evolve with environmental changes (Volff 2005). The function of cytosine is less than that of human; ammonia may not be discharged smoothly when nitrogen is excreted. Compared with salmon, the sperm capacitation of smelt may be different from that of salmon. In gained genes functional enrichment analysis, compared with human, smelt have more genes related to heparin production, which may be because the living environment of smelt is low temperature, and heparin may prevent blood coagulation. Moreover, smelt may be more sensitive to light than humans. Fish live in a different light environment from terrestrial species. However, water absorbs light, so as the water depth increases, the amount of light available decreases. Compared with zebrafish and salmon, the immune system of smelt is more complex.

\section{Transcriptome analysis}

The comparative gene expression profiles in the heat-stressed and normal groups are useful for understanding the mechanism of cellular responses under heat stress. In the HT group, when the temperature reached $23^{\circ} \mathrm{C}$, smelt began to lose population equilibrium and die, and there were no dead fish in the NT group. The liver is a vital metabolic organ that relates to stress response. In the gene enrichment analysis, we found that some genes involved in lipid metabolism, such as the lipid biosynthetic, isoprenoid metabolic, and steroid metabolic processes. Lipid is the main component of cell membranes because acute heat stress may increase the fluidity of cell membranes, and the increase in lipid metabolism may be to maintain the stability of cell membranes. Also, lipid is the main component of fat, which can block external heat, and lipid metabolism may increase the synthesis of fat to block external heat. In the Trematomus bernacchii thermal acclimation study, there was an increase in membrane saturated fatty acids (Malekar et al. 2018). Moreover, lipids may help maintain energy homeostasis in fish because lipids are the basic components of sterols. Because of the membrane's unique molecular structure, it has a thermal-sensitive macromolecular structure. Environmental stress activates lipid metabolic enzymes and targets the downstream signaling pathway (Balogh et al. 2013). Poikilothermic organisms are able to maintain their membrane 
fluidity for temperature fluctuation-induced cellular disturbance by regulating the composition of membrane lipids through physiologic and biochemical mechanisms of homeoviscous adaptation (Mendoza 2014). After heat shock, the plasma lipid peroxide level increases gradually, and severe heat stress affects the redox state and causes oxidative stress in salmon (Nakano et al. 2014). When fish suffer from hypoxia, fat metabolism is enhanced. Isoprenoid and terpenoid metabolic process-related genes, such as HMGCS1, HMGCR, and FDPS, were upregulated in liver tissue. These genes are involved in squalene synthesis. In a human cancer cell study, hypoxic cells displayed profound accumulation of squalene (Kucharzewska et al. 2015). Additionally, it plays an antioxidant role that eliminates ROS produced under stress (Micera et al. 2020). Squalene synthase is upregulated in the liver. It is the main material for the synthesis of sterols, and steroid metabolic process-related genes were upregulated in the liver under heat stress. In other studies, heat stress led to a increase in estrogen levels, and hormone disorders led to an imbalance in the number of males and females in the population (Shi et al. 2019). Some genes related to regulation of apoptotic process were significantly upregulated under heat stress. Although cells have different protective mechanisms under stress, the enhancement of stress can lead to cell signal interruption, extensive DNA damage, and cell apoptosis (Cheng et al. 2015). DNA damage is caused by hypoxia, leading to replication stress. Moreover, some genes related to regulation of cellular response to stress, such as PLRG1, RTEL1, and ING2, were upregulated. PLRG1 is involved in pre-mRNA splicing as a component of the spliceosome critical for heat environment adaptation (Huang et al. 2018). RTEL1 and ING2 play a role in DNA repair under heat stress, indicating that liver tissue has replication problems under heat stress. However, some genes related to erythrocyte characteristics, including GO term erythrocyte differentiation, erythrocyte development, and regulation of anatomical structure size, were significantly downregulated under heat stress. Erythrocytes are cellular mediators of the immune response in teleost fish; thus, the immunity of smelt is reduced under heat stress. In addition, the genes related to cell membrane signal transduction were downregulated in the heat stress group, which indicates that it is difficult for cells to communicate with extracellular components under the condition of homeostasis imbalance. Moreover, digestion-related genes have been downregulated, suggesting that abnormal temperatures lead to reduced feeding, so fish should be fed less in hot summers. One study has shown that digestive enzyme activity was significantly affected by abnormal temperature (Pimentel et al. 2015). The effect of water temperature on digestive enzymes in fish depends on species, because the optimal temperature of enzyme activity is usually in the temperature range corresponding to fish habitat (Volkoff and Rønnestad 2020). Muscle is greatly affected by heat stress because in most species, this tissue constitutes approximately $50 \%$ of the body mass. Under heat stress, apoptosis-, hypoxia-, ion transportation-, transcription-, and component organization-related genes were upregulated. In high temperatures, muscle cells lose their calcium balance, which causes muscle spasm, and in muscle contraction-related genes in which those actin-filament genes were upregulated, hypoxia can also cause muscle contraction. In an oxygen consumption study, when the water temperature rises from $14^{\circ} \mathrm{C}$ to $20^{\circ} \mathrm{C}$, the oxygen consumption of Delta smelt increased with the increase in temperature (Jeffries et al. 2016). In addition, nicotinamide adenine dinucleotide- and ubiquitination-related genes associated with cell respiration and energy metabolism were downregulated, possibly because of the decrease in collective energy metabolism caused by hypoxia.
In pathway enrichment analyses, under heat stress, the terpenoid backbone biosynthesis pathway in the liver was upregulated. The genes involved in this pathway are implicated in the final synthesis of squalene, which is an important enzyme under hypoxia conditions. Immune-related pathways, including the influenza A and Jak-STAT signaling pathways, were downregulated. Digestion-related pancreatic secretion, protein digestion, and absorption pathways were downregulated in the liver, which suggests that the digestive function of fish was disrupted under heat stress. In the muscle, several genes involved in the MAPK signaling pathway were upregulated under heat stress. A recent study has shown that the MAPK signaling pathway is activated in response to ER stress (Darling and Cook 2014). The disturbance of the ER environment, such as the decrease in $\mathrm{Ca}^{2+}$ concentration or the change in redox state, can affect protein folding and processing. Once misfolded proteins accumulate, ER stress activates a series of corresponding pathways. Under heat stress, some enriched genes in the oxytocin signaling pathway were also upregulated. Oxytocin activates the signal pathways of mRNA translation during ER stress (Klein et al. 2016). The HIF-1 signaling pathway was also upregulated in the muscle tissue, indicating that smelt suffered hypoxia stress in acute increasing temperatures. HIF1A is the main regulator of hypoxia-induced gene expression. The metabolism of smelt increases with the rise in temperature, which may lead to lack of oxygen. Smelt live in lowtemperature environments, and the oxygen solubility in lowtemperature water is higher than that in high-temperature water; thus, smelt is likely to have fewer red blood cells than temperate-water fish. Hypoxic stress- induced unfolded protein response (UPR) and autophagy. Hypoxia causes perturbations in ER activity, resulting in UPR activation. These upregulated pathways suggested that smelt suffered hypoxia and ER stress under heat stress. ER stress-related genes NFE2L1 and ERGIC1 were upregulated in the muscle tissue. ER stress was also observed under heat stress in Atlantic salmon (Shi et al. 2019). The IBD, Leishmaniasis, African trypaosomiasis, and type 1 diabetes pathways were downregulated in muscle under heat stress; these are related to immune and infectious diseases, considering that heat stress has a negative effect on the inner immune system (Lyu et al. 2018).

\section{Conclusions}

Using long reads and short reads from the PacBio Sequel and NovaSeq6000 platforms, respectively, we successfully assembled the draft genome and obtained the first reference genome of $H$. nipponensis. In transcriptome analysis, smelt suffer from hypoxia and ER stress, which leads to severe oxidative stress in the body under heat stress. These results provide a better understanding of the molecular mechanisms regulating the response of H. nipponensis under heat stress, which will help prevent and treat damage to fish caused by high water temperature.

\section{Funding}

This research was supported by a grant from the Collaborative Genome Program of the Korea Institute of Marine Science and Technology Promotion funded by the Ministry of Oceans and Fisheries (No. 20180430) and the National Institute of Fisheries Science (R2021038). Biao Xuan was supported by the BK21 Plus Program from Ministry of Education. 


\section{Data availability}

H. nipponensis genome assembly was deposited in NCBI with accession number of SAMN16577099. RNA-Seq raw sequences were deposited in the Short Read Archive of the NCBI with accession number PRJNA672783. Supplementary material is available at figshare: https://doi.org/10.25387/g3.14489730.

\section{Conflicts of interest}

None declared.

\section{Literature cited}

Balogh G, Péter M, Glatz A, Gombos I, Török Z, et al. 2013. Key role of lipids in heat stress management. FEBS Lett. 587:1970-1980. doi: 10.1016/j.febslet.2013.05.016.

Basu N, Basu N, Todgham AE, Todgham AE, Ackerman PA, et al. 2002. Heat shock protein genes and their functional significance in fish. Gene. 295:173-183.

Cheng CH, Yang FF, Liao SA, Miao YT, Ye CX, et al. 2015. High temperature induces apoptosis and oxidative stress in pufferfish (Takifugu obscurus) blood cells. J Therm Biol. 53:172-179. doi: 10.1016/j.jtherbio.2015.08.002

Choi S, Kim EB. 2019. Complete mitochondrial genome sequence and SNPs of the Korean smelt Hypomesus nipponensis (Osmeriformes, Osmeridae). Mitochondrial DNA Part B Res. 4:1844-1845. doi: 10.1080/23802359.2019.1613178.

Darling NJ, Cook SJ. 2014. The role of MAPK signalling pathways in the response to endoplasmic reticulum stress. Biochim Biophys Acta. 1843:2150-2163. doi:10.1016/j.bbamcr.2014.01.009.

Fan G, Song Y, Yang L, Huang X, Zhang S, et al. 2020. Initial data release and announcement of the 10,000 Fish Genomes Project (Fish10K). GigaScience. 9. doi:10.1093/gigascience/giaa080.

Fulda S, Gorman AM, Hori O, Samali A. 2010. Cellular stress responses: cell survival and cell death. Int J Cell Biol. 2010: 214074. doi:10.1155/2010/214074.

Haas BJ, Papanicolaou A, Yassour M, Grabherr M, Blood PD, et al. 2013. De novo transcript sequence reconstruction from RNA-seq using the Trinity platform for reference generation and analysis. Nat Protoc. 8:1494-1512. doi:10.1038/nprot.2013.084.

Huang J, Li Y, Liu Z, Kang Y, Wang J. 2018. Transcriptomic responses to heat stress in rainbow trout Oncorhynchus mykiss head kidney. Fish Shellfish Immunol. 82:32-40. doi:10.1016/j.fsi.2018.08.002.

Jeffries KM, Connon RE, Davis BE, Komoroske LM, Britton MT, et al. 2016. Effects of high temperatures on threatened estuarine fishes during periods of extreme drought. J Exp Biol. 219:1705-1716. doi: 10.1242/jeb.134528.

Kelly T, Johnsen H, Burgerhout E, Tveiten H, Thesslund T, et al. 2020. Low oxygen stress during early development influences regulation of hypoxia-response genes in farmed Atlantic salmon (Salmo salar). G3 (Bethesda). 10:3179-3188. doi:10.1534/g3.120.401459.

Kim EB, Fang X, Fushan AA, Huang Z, Lobanov AV, et al. 2011. Genome sequencing reveals insights into physiology and longevity of the naked mole rat. Nature. 479:223-227. doi:10.1038/nature10533.

Klein BY, Tamir H, Hirschberg DL, Ludwig RJ, Glickstein SB, et al. 2016. Oxytocin opposes effects of bacterial endotoxin on ER-stress signaling in Caco2BB gut cells. Biochim Biophys Acta. 1860:402-411. doi:10.1016/j.bbagen.2015.10.025.
Kucharzewska P, Christianson HC, Belting M. 2015. Global profiling of metabolic adaptation to hypoxic stress in human glioblastoma cells. PLoS One. 10:e0116740.doi:10.1371/journal.pone.0116740.

Logan CA, Buckley BA. 2015. Transcriptomic responses to environmental temperature in eurythermal and stenothermal fishes. J Exp Biol. 218:1915-1924. doi:10.1242/jeb.114397.

Lu Y, Wu Z, Song Z, Xiao P, Liu Y, et al. 2016. Insight into the heat resistance of fish via blood: effects of heat stress on metabolism, oxidative stress and antioxidant response of olive flounder Paralichthys olivaceus and turbot Scophthalmus maximus. Fish Shellfish Immunol. 58:125-135. doi:10.1016/j.fsi.2016.09.008.

Lyu L, Wen H, Li Y, Li J, Zhao J, et al. 2018. Deep transcriptomic analysis of black rockfish (Sebastes schlegelii) provides new insights on responses to acute temperature stress. Sci Rep. 8. doi: 10.1038/s41598-018-27013-z.

Malekar VC, Morton JD, Hider RN, Cruickshank RH, Hodge S, et al. 2018. Effect of elevated temperature on membrane lipid saturation in Antarctic notothenioid fish. PeerJ. 6:e4765. doi:10.7717/peerj.4765.

Malmstrøm M, Matschiner M, Tørresen OK, Star B, Snipen LG, et al. 2016. Evolution of the immune system influences speciation rates in teleost fishes. Nat Genet. 48:1204-1210. doi:10.1038/ng.3645.

Mendoza DD. 2014. Temperature sensing by membranes. Annu Rev Microbiol. 68:101-116. doi:10.1146/annurev-micro-091313-103612.

Micera M, Botto A, Geddo F, Antoniotti S, Bertea CM, et al. 2020 Squalene: more than a step toward sterols. Antioxidants. 9: 688-614. doi:10.3390/antiox9080688.

Moriya Y, Itoh M, Okuda S, Yoshizawa AC, Kanehisa M. 2007. KAAS: an automatic genome annotation and pathway reconstruction server. Nucleic Acids Res. 35:W182-W185. doi: 10.1093/nar/gkm321.

Nakano T, Kameda M, Shoji Y, Hayashi S, Yamaguchi T, et al. 2014. Effect of severe environmental thermal stress on redox state in salmon. Redox Biol. 2:772-776. doi:10.1016/j.redox.2014.05.007.

Narum SR, Campbell NR. 2015. Transcriptomic response to heat stress among ecologically divergent populations of redband trout. BMC Genomics. 16. doi:10.1186/s12864-015-1246-5.

Olsvik PA, Vikeså V, Lie KK, Hevrøy EM. 2013. Transcriptional responses to temperature and low oxygen stress in Atlantic salmon studied with next-generation sequencing technology. BMC Genomics. 14:817. doi:10.1186/1471-2164-14-817.

Pimentel MS, Faleiro F, Diniz M, Machado J, Pousão-Ferreira P, et al. 2015. Oxidative stress and digestive enzyme activity of flatfish larvae in a changing ocean. PLoS One. 10:e0134082. doi: 10.1371/journal.pone.0134082.

Qiang J, Bao WJ, Tao FY, He J, Li XH, et al. 2017. The expression profiles of miRNA-mRNA of early response in genetically improved farmed tilapia (Oreochromis niloticus) liver by acute heat stress. Sci Rep. 7:1-15. doi:10.1038/s41598-017-09264-4.

Robinson MD, McCarthy DJ, Smyth GK. 2010. edgeR: a Bioconductor package for differential expression analysis of digital gene expression data. Bioinformatics. 26:139-140. doi:10.1093/bioinformatics/btp616.

Saruwatari T, Lopez JA, Pietsch TW. 1997. A revision of the Osmerid genus Hypomesus Gill (Teleostei: Salmoniformes), with the description of a new species from the Southern Kuril Islands. SpecDiv. 2:59-82. doi:10.12782/specdiv.2.59.

Shi KP, Dong SL, Zhou YG, Li Y, Gao QF, et al. 2019. RNA-seq reveals temporal differences in the transcriptome response to acute heat stress in the Atlantic salmon (Salmo salar). Comp Biochem Physiol Part D Genomics Proteomics. 30:169-178. doi: 10.1016/j.cbd.2018.12.011. 
Simão FA, Waterhouse RM, Ioannidis P, Kriventseva EV, Zdobnov EM. 2015. BUSCO: assessing genome assembly and annotation completeness with single-copy orthologs. Bioinformatics. 31: 3210-3212. doi:10.1093/bioinformatics/btv351.

Volff JN. 2005. Genome evolution and biodiversity in teleost fish. Heredity (Edinb). 94:280-294. doi:10.1038/sj.hdy.6800635.

Volkoff H, Rønnestad I. 2020. Effects of temperature on feeding and digestive processes in fish. Temperature (Austin). 7:307-320. doi: 10.1080/23328940.2020.1765950.

Von Schalburg KR, Leong J, Cooper GA, Robb A, Beetz-Sargent MR, et al. 2008. Rainbow smelt (Osmerus mordax) genomic library and
EST resources. Mar Biotechnol (NY). 10:487-491. doi: 10.1007/s10126-008-9089-6.

Walker BJ, Abeel T, Shea T, Priest M, Abouelliel A, et al. 2014. Pilon: an integrated tool for comprehensive microbial variant detection and genome assembly improvement. PLoS One. 9:e112963. doi: 10.1371/journal.pone.0112963.

Yu G, Wang LG, Han Y, He QY. 2012. ClusterProfiler: an R package for comparing biological themes among gene clusters. OMICS. 16: 284-287. doi:10.1089/omi.2011.0118.

Communicating editor: B. Andrews 